\title{
A tomografia computadorizada de feixe cônico como exame complementar na Endodontia: relato de caso
}

Cone-beam computed tomography as a complementary examination in Endodontics: case report Tomografía computarizada de haz cónico como examen complementario en Endodoncia: reporte de caso Caroline Mentor Andrade GALVÃO ${ }^{1}$ Pedro José Targino RIBEIRO ${ }^{2}$

Gabriella de Vasconcelos NEVES ${ }^{3}$ Diego Filipe Bezerra SILVA ${ }^{2}$ Gustavo Araújo de FREITAS ${ }^{4}$ Daliana Queiroga de Castro GOMES ${ }^{5}$

${ }^{I}$ Graduada em Odontologia pela Universidade Estadual da Paraíba - UEPB, 58233-000, Araruna-PB, Brasil

${ }^{2}$ Mestrando do Programa de Pós-Graduação em Odontologia da Universidade Estadual da Paraíba - UEPB, 58429-600, Campina Grande-PB, Brasil

${ }^{3}$ Mestranda do Programa de Pós-Graduação em Odontologia da Universidade Estadual da Paraíba - UEPB, 58429-600, Campina Grande-PB, Brasil ${ }^{4}$ Graduando do curso de Odontologia da Universidade Estadual da Paraíba - UEPB, 58429-600, Campina Grande-PB, Brasil

${ }_{5}^{5}$ Professora Doutora do Programa de Pós-Graduação em Odontologia da Universidade Estadual da Paraíba - UEPB, 58429-600, Campina Grande-PB, Brasil

\section{Resumo}

Introdução: A tomografia computadorizada de feixe cônico (TCFC) consiste em um moderno sistema de imagens radiográficas criado especificamente para a análise do complexo maxilofacial. Devido às limitações apresentadas pelas radiografias convencionais, esse exame mostra-se de grande importância para o diagnóstico endodôntico, pois apresenta diversas vantagens como melhor qualidade de imagem, reconstrução tridimensional e as doses de radiação mais baixas. Objetivo: Enfatizar, por meio de um relato de caso, a importância da TCFC como método auxiliar de diagnóstico na localização de canais radiculares. Relato de caso: Paciente do sexo feminino, 25 anos de idade, procurou atendimento odontológico com queixa de dor à mastigação no dente 14, que já havia sido tratado endodonticamente há um ano e retratado há seis meses. Após realização de radiografia periapical, observou-se uma imagem radiolúcida envolvendo o ápice do dente 14. Diante disso, realizou-se uma TCFC, a qual revelou a presença de um canal não obturado. Com bases nos achados, optou-se por uma nova intervenção endodôntica em duas sessões, sendo que, na segunda sessão, a paciente encontrava-se assintomática e sem edema na região. Após um ano de proservação, a paciente não relatou sintomatologia dolorosa e nenhum sinal de infecção foi observado. Conclusão: Dessa forma, foi possível concluir que a TCFC constituiu-se como um método preciso para auxiliar no diagnóstico, pois permitiu a visualização de um canal radicular não instrumentado, além de permitir um melhor direcionamento do plano de tratamento.

Descritores: Tomografia Computadorizada de Feixe Cônico; Endodontia; Complicações Intraoperatórias.

\section{Abstract}

Introduction: Cone-beam computed tomography (CBCT) is a modern radiographic imaging system created specifically for the analysis of the maxillofacial skeleton. Due to the limitations presented by conventional radiographs, this examination is of great importance for endodontic diagnosis, as it has several advantages such as better image quality, three-dimensional reconstruction and lower radiation doses. Objetivo: Enfatizar, por meio de um relato de caso, a importância da TCFC como método auxiliar de diagnóstico na localização de canais radiculares. Objective: To emphasize, through a case report, the importance of CBCT as an auxiliary diagnostic method in the localization of root canals. Case report: A 25-year-old female patient sought dental care complaining of chewing pain on tooth 14, which had already been endodontically treated for one year and was portrayed six months ago. After periapical radiography, a radiolucent image involving the apex of the tooth was observed 14. Upon this, a CBCT was performed, which revealed the presence of an unfilled canal. Based on the findings, we opted for a new endodontic intervention in two sessions, where, in the second session, the patient was asymptomatic and without edema in the region. After one year of proservation, the patient reported no painful symptoms and no signs of infection were observed. Conclusion: Thus, it was possible to conclude that CBCT was an accurate method to aid the diagnosis, as it allowed the visualization of an uninstructed root canal, as well as allowing a better direction of the treatment plan.

Descriptors: Cone-beam Computed Tomography; Endodontics; Intraoperative Complications.

\section{Resumen}

Introducción: La tomografía computarizada de haz cónico (TCHC) es un moderno sistema de imágenes radiográficas creado específicamente para el análisis del complexo maxilofacial. Debido a las limitaciones que presentan las radiografías convencionales, este examen es de gran importancia para el diagnóstico endodóntico, ya que tiene varias ventajas, como una mejor calidad de imagen, reconstrucción tridimensional y dosis de radiación más bajas. Objetivo: Enfatizar, a través de um reporte de caso, la importancia de TCHC como método de diagnóstico auxiliar en la localización de los conductos radiculares. Reporte del caso: Una paciente de 25 años buscó atención dental quejándose de dolor de masticación en el diente 14, que ya había sido tratado endodónticamente durante un año y fue retratado hace seis meses. Después de la radiografía periapical, se observó una imagen radiolúcida que involucra el ápice del diente 14. Tras esto, se realizó una TCHC, que reveló la presencia de un canal sin relleno. Con base en los hallazgos, optamos por una nueva intervención endodóntica en dos sesiones, donde, en la segunda sesión, el paciente estaba asintomático y sin edema en la región. Después de un año de conservación, el paciente no informó síntomas dolorosos y no se observaron signos de infección. Conclusión: Por lo tanto, fue posible concluir que la CBCT era un método preciso para ayudar al diagnóstico, ya que permitía la visualización de un conducto radicular no instruido, además de permitir una mejor dirección del plan de tratamiento.

Descriptores: Tomografía computarizada de Haz Cónico; Endodoncia; Complicaciones Intraoperatorias.

\section{INTRODUÇÃO}

O sucesso do tratamento endodôntico requer um conhecimento preciso da morfologia dos canais radiculares e das suas possíveis variações, que influenciam diretamente na qualidade do desbridamento, desinfecção e obturação dos mesmos. O sistema de canais radiculares pode ser complexo e difícil de avaliar, e por vezes canais secundários e acessórios estão imperceptíveis clinicamente, acarretando infecções secundárias. Por essa razão, faz-se necessário o entendimento das suas múltiplas variações anatômicas, seja sob condições normais ou não, reduzindo assim as chances de falha durante procedimentos endodônticos ${ }^{1-3 .}$

Desde a sua criação, a radiografia convencional tem permanecido como a base da imaginologia em Endodontia. Nas últimas décadas, no entanto, avanços na imaginologia médica têm sido aplicados, com variável sucesso, nas diversas áreas 
da odontologia, propiciando tratamentos mais seguros e eficazes ${ }^{2}$.

O exame radiográfico é um componente essencial no tratamento endodôntico que aponta aspectos do diagnóstico, planejamento do tratamento endodôntico, controle intraoperatório e avaliação dos resultados fornecendo informações úteis para a presença e localização de lesões perirradiculares, anatomia do canal radicular e a proximidade de estruturas anatômicas adjacentes ${ }^{4}$. Entretanto, as radiografias periapicais apresentam suas limitações para avaliação da anatomia radicular, devido à sobreposição das raízes ou de suas estruturas circundantes, por proporcionar uma imagem bidimensional de uma estrutura tridimensional. Em decorrência disso, a tomografia computadorizada de feixe cônico (TCFC) ganhou espaço e se tornou um recurso complementar importante para o diagnóstico endodôntico. Por se tratar de um método seguro e preciso para avaliar o sistema de canais radiculares ${ }^{4,5}$.

A TCFC foi inserida no campo da endodontia em 1990. A técnica usa uma fonte de radiação em forma de cone para adquirir imagens dados em um arco de rotação completo ou parcial, exibindo o contorno tridimensional de um objeto, permitindo assim ao clínico conseguir uma representação mais realista das estruturas a serem estudadas. A capacidade da TCFC de reduzir ou eliminar a sobreposição das estruturas vizinhas a torna superior às radiografias periapicais ${ }^{3}$.

$\mathrm{Na}$ Odontologia, é de grande importância a avaliação tridimensional das estruturas dentais e tecidos associados, porém a limitação das radiografias convencionais pode ser considerada um problema. Diante do exposto, o objetivo deste trabalho é relatar e discutir a importância do uso da TCFC como exame auxiliar na endodontia, levantando informações relevantes por meio de um relato de caso.

\section{CASO CLÍNICO}

Paciente, sexo feminino, 25 anos de idade, procurou atendimento odontológico com queixa de dor à mastigação no dente 14 , que já havia sido tratado endodonticamente há um ano. Foi realizada uma anamnese detalhada a fim de se obter todas as informações acerca da queixa principal, história médica e odontológica. A história médica pregressa não revelou nenhuma informação relevante. Segundo relato da paciente, após seis meses do primeiro tratamento, a sintomatologia dolorosa persistiu, e o dente foi submetido a um retratamento endodôntico, porém, sem sucesso na regressão da dor. Ao exame físico intraoral, observou-se discreto edema na região vestibular. $\mathrm{O}$ teste térmico de sensibilidade pulpar e o teste de percussão vertical e horizontal foram realizados, ambos com respostas negativas. Por meio de uma radiografia periapical convencional, foi possível observar uma imagem radiolúcida envolvendo o ápice do dente 14 (Figura 1). Diante do caso, notou-se a necessidade da solicitação de uma tomografia computadorizada por feixe cônico para uma melhor avaliação anatômica do dente em questão. Por meio do exame tomográfico de feixe cônico, foi possível observar a presença de um canal não obturado (Figura 2), o qual provavelmente foi a causa da persistência do quadro doloroso e do surgimento da lesão periapical.

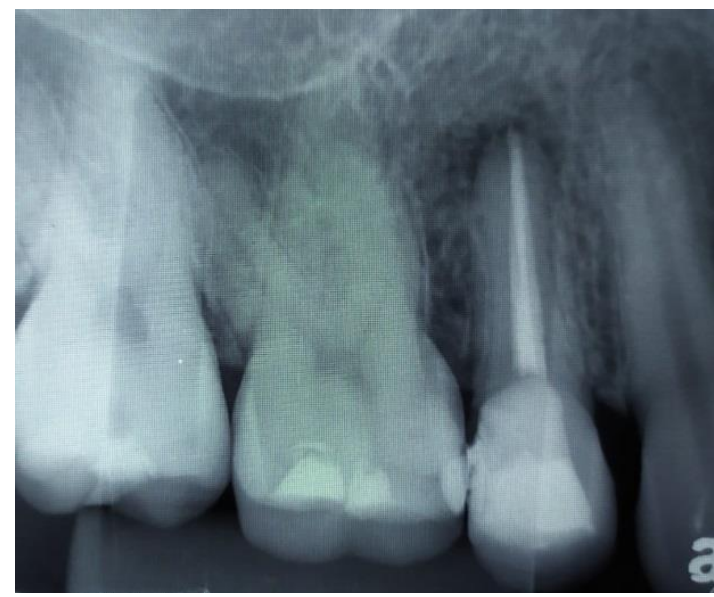

Figura 1: Aspecto radiográfico inicial do dente 14, observado por meio de uma radiografia periapical convencional.

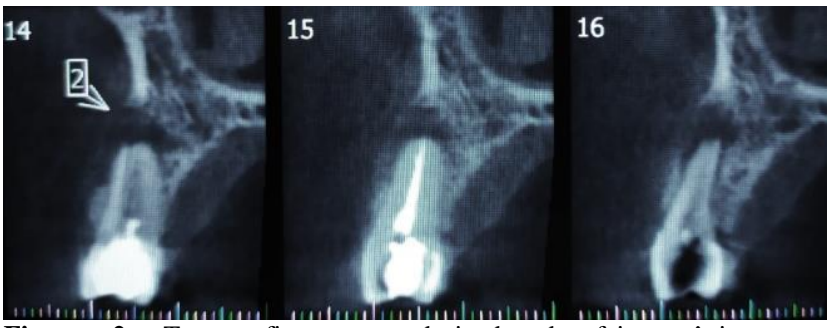

Figura 2: Tomografia computadorizada de feixe cônico com reconstruções parassagitais.

Com bases nos achados, optou-se pela realização de uma nova intervenção endodôntica em duas sessões. Na primeira sessão, foi realizada a desobturação do canal vestibular com auxílio dos alargadores Gates Glidden (Dentsply-Maillefer, Ballaigues, Suiça) no terço cervical e do instrumento Reciproc ${ }^{\circ}$ (VDW, Munique, Alemanha) R25 nos terços médio e apical. Após completa desobturação, constatada por meio de uma radiografia periapical, deu-se sequência à instrumentação do canal vestibular com auxilío do instrumento Reciproc $®$ R40. Em seguida, o canal palatino foi identificado e instrumentado até o diâmetro cirúrgico de $0,40 \mathrm{~mm}$, por meio do instrumento Reciproc ${ }^{\circledR}$ R40. A cada troca de instrumento, foi realizada irrigação, aspiração e inundação com hipoclorito de sódio $(\mathrm{NaOCl})$ a $2,5 \%$ (Asfer®, Santa Maria, SP) por meio das agulhas NaviTip ${ }^{\circledR}$ (Ultradent, South Jordan, UT, EUA) utilizando Kit de irrigação-aspiração Ultradent ${ }^{\circledR}$ (Ultradent, South Jordan, UT, EUA).

Após o término do preparo químicomecânico, deu-se sequência ao protocolo de agitação ultrassônica dos canais radiculares, com três ciclos de 20 segundos, alternando as soluções químicas 
(Hipoclorito de sódio/ EDTA/ Hipoclorito de sódio). O inserto utilizado foi o Irrisonic E1 (Helse Dental Technology ${ }^{\circledR}$, SP, Brasil) acoplado em ultrassom (JetSonic BP, Gnatus ${ }^{\circledR}$, Ribeirão Preto, SP, Brasil) com a ponta posicionada a $1,0 \mathrm{~mm}$ aquém do comprimento real de trabalho. Os canais foram secos com o auxílio de pontas de papel absorvente estéreis de calibre 40 (Dentsply-Maillefer, Ballaigues, Suiça). Foi utilizado como medicação intracanal o hidróxido de cálcio (UltraCal XS; Ultradent, South Jordan, UT) e a coroa foi selada com ionômero de vidro Restaurador Riva Light Cure ${ }^{\circledR}$ (SDI, Victoria, Austrália). Na sessão seguinte, após 21 dias, a paciente encontrava-se assintomática, e foi possível observar ausência de edema e sensibilidade à percussão. Optou-se, pela obturação dos canais radiculares pela técnica do cone único. Ao final do procedimento, foi realizada uma radiografia pericapical, que mostrou selamento adequado dos canais radiculares (Figura 3). A paciente não relatou episódios de dor ou desconforto e nenhum sinal de infecção foi observado após um ano de acompanhamento.

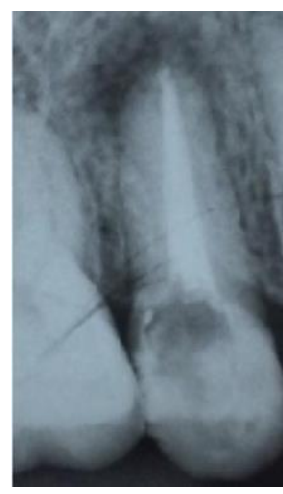

Figura 3: Aspecto radiográfico imediato após retratamento endodôntico.

DISCUSSÂO

O tratamento dos canais radiculares é um procedimento odontológico comum para preservar os dentes, tratando doenças da polpa dentária e região perirradicular ${ }^{6}$. A terapia endodôntica consiste principalmente na limpeza quimico-mecânica do sistema de canais radiculares, de modo a remover todos os detritos de tecido mole do interior do dente e sua completa obturação tridimensional com um material inerte ${ }^{7-11}$.

Os principais motivos para a falha endodôntica são instrumentação incompleta dos canais radiculares e a presença de canais não $\operatorname{tratados}^{7,8}$. No caso ora relatado, a paciente continuava apresentando sintomatologia dolorosa no dente 14, mesmo após um retratamento endodôntico, pois um dos canais radiculares havia sido identificado e tratado endodonticamente, como verificado no exame de TCFC.

$\mathrm{O}$ tratamento endodôntico bem-sucedido e previsível requer conhecimento da biologia, anatomia dos canais radiculares ${ }^{6,10-15}$ e avaliação radiográfica cuidadosa para determinar o número de raízes e canais radiculares. ${ }^{12}$ A morfologia dos canais radiculares varia muito entre diversas populações e até mesmo entre indivíduos dentro da mesma população ${ }^{16}$.

As raízes dos primeiros pré-molares superiores diferem de outros pré-molares, apresentando uma variedade de configurações e formas em toda a dentição ${ }^{17}$. Dessa forma, o primeiro pré-molar maxilar representa um dos dentes mais difíceis de serem tratados endodonticamente ${ }^{16}$. Em revisão da literatura com objetivo de revisar os estudos anatômicos e casos clínicos que relataram a anatomia interna e externa dos primeiros pré-molares superiores, foi observado que, a partir da análise de 92 estudos, a maioria dos primeiros pré-molares superiores tinha uma raiz $(41,7 \%)$ ou duas raízes $(56,6 \%)$. Independentemente do número de raízes, a maioria $(86,6 \%)$ dos dentes tinha dois canais radiculares ${ }^{9}$. No presente caso, o dente da paciente apresentava apenas uma raiz e dois canais radiculares, vestibular e palatino, separados e distintos.

Recentemente, em estudo realizado por de Lima et al. ${ }^{18}$, com objetivo de avaliar a configuração do canal radicular de pré-molares superiores usando 999 tomografias computadorizadas de feixe cônico (TCFC) de uma subpopulação do sudeste brasileiro, foi observado que $82,2 \%$ dos primeiros pré-molares analisados possuíam 2 canais radiculares separados e distintos, apesar de que apenas 16,7\% desses dentes, quando unirradiculares, possuía essa mesma configuração $^{18}$. Em consonância, no estudo de Li et al. ${ }^{6}$ (2018) com pacientes chineses, $83,1 \%$ dos pacientes apresentaram primeiros pré-molares superiores com uma raíz e dois canais, sendo que $19 \%$ desses dentes unirradiculares apresentaram dois canais separados e distintos.

A avaliação radiográfica odontológica é uma ferramenta fundamental para auxiliar no diagnóstico endodôntico ${ }^{7,19,20}$. Radiografias periapicais intraorais convencionais são rotineiramente empregadas para avaliar a anatomia dos canais radiculares ${ }^{7,19}$. A utilização das radiografias em ângulo mesial ou distal podem ajudar a determinar o número de raízes dos dentes $^{12}$. No entanto, essas radiografias possuem uma limitação inerente, ou seja, fornecem uma visão bidimensional de uma estrutura tridimensional, o que leva à distorção geométrica e, consequentemente, interpretação errônea e / ou diagnóstico errôneo ${ }^{19,20}$. No presente caso, o tratamento endodôntico inicial foi realizado utilizando-se a radiografia periapical como exame auxiliar na identificação dos condutos, porém sem sucesso, o que comprovou a limitação da radiografia periapical e a necessidade da realização da TCFC.

Com o desenvolvimento da técnica da TCFC, tornou-se possível estudar a anatomia dos dentes com mais detalhes, devido às imagens tridimensionais de 
alta qualidade que podem ser obtidas a partir dessa técnica $^{14}$, sem sobreposição de estruturas ${ }^{8,18}$. Suas aplicações intraoperatórias incluem localização de canais calcificados, avaliação de achados anatômicos inesperados, avaliação de canais perdidos em retratamento endodôntico, avaliação de reabsorção radicular e fraturas radiculares e avaliação de erros iatrogênicos como perfuração, instrumentos fraturados e sobreobturação ${ }^{19}$. No caso ora relatado, houve a necessidade de solicitar uma TCFC porque apesar de ter sido realizado retratamento endodôntico no dente referido anteriormente, havia ainda sintomatologia dolorosa.

Apesar das vantagens da TCFC, deve-se evitar utilizá-la como exame complementar de rotina na Endodontia. Os profissionais devem ter conhecimento prévio da anatomia das raízes, bem como das variações anatômicas dos canais radiculares para obter sucesso na terapia endodôntica. Além disso, deve-se recorrer a angulações nas tomadas radiográficas para se observar possíveis canais radiculares que seriam sobrepostos numa tomada radiográfica convencional.

\section{CONSIDERAÇÕES FINAIS}

Pode-se concluir que a imagem tomográfica de feixe cônico forneceu uma informação diagnóstica mais precisa comparada à radiografia periapical convencional, e que essa informação influenciou diretamente as estratégias de retratamento endodôntico.

\section{REFERÊNCIAS}

1. Durack C, Patel S. Cone beam computed tomography in endodontics. Braz Dent J. 2012;23(3):179-91.

2. Falcão CA, Albuquerque VC, Amorim NL, Freitas SA, Santos TC, Matos FT, Ferraz MA. Frequency of the mesiopalatal canal in upper first permanent molars viewed through computed tomography. Acta Odontol Latinoam. 2016;29(1):54-9.

3. Dalili Kajan Z, Taramsari M, Khosravi Fard N, Kanani M. Accuracy of Cone-beam Computed Tomography in Comparison with Standard Method in Evaluating Root Canal Morphology: An In Vitro Study. Iran Endod J. 2018; 13(2):181-87.

4. Cardona-Castro JA; Fernández-Grisaies R. Root anatomy, a view from the microsurgery endodontic: review. CES odontol. 2015;28(2):70-99.

5. Aminoshariae A, Kulild JC, Syed A. Cone-beam computed tomography compared with intraoral radiographic lesions in endodontic outcome studies: a systematic review. J Endod. 2018;44(11):1626-31.

6. Li YH, Bao SJ, Yang XW, Tian XM, Wei B, Zheng YL. Symmetry of root anatomy and root canal morphology in maxillary premolars analyzed using cone-beam computed tomography. Arch Oral Biol. 2018;94:84-92.

7. Rajasekhara S, Sharath Chandra S, Parthasarathy LB. Cone beam computed tomography evaluation and endodontic management of permanent mandibular second molar with four roots: A rare case report and literature review. J Conserv Dent. 2014;17(4):385-88.

8. Shetty A, Hegde MN, Tahiliani D, Shetty H, Bhat GT, Shetty S. A three-dimensional study of variations in root canal morphology using conebeam computed tomography of mandibular premolars in a South Indian population. J Clin Diagn Res. 2014;8(8):ZC22-4.

9. Ahmad IA, Alenezi MA. Root and Root Canal Morphology of Maxillary First Premolars: A Literature Review and Clinical Considerations. J Endod. 2016;42(6):861-72.

10. Sagale AA, Ramugade MM, Ganga R. Endodontic Management of Mandibular Second Premolar with Type IX Canal Configuration Using Cone-Beam Computed Tomography as a Diagnostic Aid: A Rare Case Report. Contemp Clin Dent. 2018;9(Suppl 1):S180-84.

11. Martins JNR, Gu Y, Marques D, Francisco H, Caramês J. Differences on the Root and Root Canal Morphologies between Asian and White Ethnic Groups Analyzed by Cone-beam Computed Tomography. J Endod. 2018; 44(7):1096-104.

12. Balakasireddy K, Kumar KP, John G, Gagan C. Cone Beam Computed Tomography Assisted Endodontic Management of a Rare Case of Mandibular First Premolar with Three Roots. J Int Oral Health. 2015;7(6):107-9.

13. Ugur Z, Akpinar KE, Altunbas D. Maxillary first premolars with three root canals: two case reports. J Istanb Univ Fac Dent. 2017;51(3):50-4.

14. Razumova S, Brago A, Khaskhanova L, Howijieh A, Barakat H, Manvelyan A. A Cone-Beam Computed Tomography Scanning of the Root Canal System of Permanent Teeth among the Moscow Population. Int J Dent. 2018; 2018:2615746.

15. Pan JYY, Parolia A, Chuah SR, Bhatia S, Mutalik S, Pau A. Root canal morphology of permanent teeth in a Malaysian subpopulation using conebeam computed tomography. BMC Oral Health. 2019 Jan 14;19(1):14.

16. Senan EM, Alhadainy HA, Genaid TM, Madfa AA. Root form and canal morphology of maxillary first premolars of a Yemeni population. BMC Oral Health. 2018;18(1):94.

17. Fan L, Yuan K, Niu C, Ma R, Huang Z. A conebeam computed tomography study of the mesial cervical concavity of maxillary first premolars. Arch Oral Biol. 2018;92:79-82.

18. de Lima CO, de Souza LC, Devito KL, do Prado 
M, Campos CN. Evaluation of root canal morphology of maxillary premolars: a cone-beam computed tomography study. Aust Endod J. 2019;45(2):196-201.

19. Ball RL, Barbizam JV, Cohenca N. Intraoperative endodontic applications of cone-beam computed tomography. J Endod. 2013;39(4):548-57.

20. Kaur A, Logani A. Usefulness of cone beam computed tomography in perplexing endodontic cases. Endodontology. 2018;30:187-93.

\section{CONFLITO DE INTERESSES}

Os autores declaram não haver conflitos de interesse.

\section{AUTOR PARA CORRESPONDÊNCIA}

Daliana Queiroga de Castro Gomes

Departamento de Odontologia

Universidade Estadual da Paraíba, UEPB

Rua Baraúnas, 351, Bairro Universitário

58429-500 Campina Grande- PB, Brasil

Email: dqcgomes@hotmail.com

Submetido em 24/08/2019

Aceito em 23/09/2020 